\title{
THE LANDSLIDE HAZARD MAP OF BOGOTA AN UPDATING
}

\author{
N. J. Zamora \\ Risk management and climate change Institute, Bogotá, Colombia - \\ nzamora.idiger@gmail.com
}

Commission IV, WG IV/4

KEYWORDS: Landslides, Bogota, GRASS GIS, Risk management, Modelling, Model Builder

\begin{abstract}
:
A land management plan (Plan de Ordenamiento Territorial, POT) is an instrument for planning the land-use of a territory considering physical, social and economic aspects. In the frame of the POT's upgrading, the risk management plays an important role in conservation of human lives, prioritization of territory's land-use as well as the formulation to reduce the fiscal vulnerability of the territorial institutions when a disaster occurs e.g. landslides. Landslides represent a rampant danger in Bogota's hillside area because of accelerated urban growth, steep topography, degraded rocks, susceptible soils and abundant rain among other factors. It is calculated that more than 3500000 people live in such risky zones.

This paper presents the methodology followed to update the landslide hazard map -from the GIS techniques perspective produced by an interdisciplinary team formed by geologists, geotechnical and GIS specialists.

The methodology used to obtain the map of landslide hazards consists of the combination of two partial results regarding to the evaluation and analysis of slopes' stability in the studied area. The first one has to with a heuristic method that combines the variables prone to influence the occurrence of landslides. The following eight variables were included: relief, drainage, and material, land use as susceptibility factors and erosion, climate, earthquake, human factor as triggering factors. To combine these variable map algebra was used. While the second partial result involves stability of natural slopes.

Despite the fact that in Latin-American region and especially in public institutions proprietary software is widely used, the visualisation and edition of geographic information was performed with QGIS software. Additionally, the study area covered a considerable part of Bogota's urban region which demanded a quite efficient and robust software that allow to execute a straightforward and complex processing. GRASS GIS software was used to carry out most of the geoprocessing tasks without splitting up the datasets. The Open Source GRASS yielded excellent results proving be an efficient tool when dealing with big datasets. Furthermore, in the study of some variables very specific geomorphology algorithms, such as Terrain Classification from SAGA GIS, were used. Meanwhile automation of some geoprocesses was implemented with proprietary software.

New techniques and procedures as well as detailed inputs with further level of detail provided a more accurate map in a shorter period of time. One of this techniques is the delimitation of areas for evaluating the stability of natural earth slopes which was analysed with the support of hydrological modelling in GIS. This helped to guarantee the homogeneity of these regions, yielding quite acceptable results when accuracy is proved as an important condition in the application of this method.

This map constitutes a valuable input in the risk management when dealing with land planning of the city. From this new hazard map, new suitable areas for housing can be defined. Also, it is the initial input for determining the necessity of new civil works for disaster mitigation. In some cases, resettlement of affected people will be recommended when the levels of landslide's hazards are not capable of being mitigated.
\end{abstract}

\section{INTRODUCTION}

The current POT and its corresponding landslide hazard map were released in 2000. The dynamic of the landscape due to natural and human events lead to an imperative necessity of updating the landslide hazard map of Bogota. For configuring the new map a $1 \mathrm{~m}$ resolution DEM was used as one of the most important inputs. In addition, Orthophotos and Google Earth were reviewed. Conversely, specific open source was used to carry out some geoprocessing tasks yielding more precise results. The current hardware capacity eased the work allowing producing a map in a short period of time. Utilisation of more detailed outputs as well as new GIS techniques played an important role in this updating.

Moreover, hydrological modelling granted a more accurate slope's family delimitation and a Visual Basic application developed to find the main inputs for the
Natural Slopes Methodology (NSM) made the process expedite and provided reliable results.

The resulting map is derived from a model that has into account different factors and combines statistical (SSE Semiquantitative Stability Evaluation) with probabilistic methods (NSM Natural Slopes Methodology). Like in every model a simplification of reality is made selecting the most relevant aspects that affect an event in this case stability of slopes. An appropriate way for validating the model is comparing the known hazard levels of morphodynamic processes located in the study's area with those ones yielded by the map. If they produce an acceptable level of adjustment, the model will give satisfactory results and will be adopted.

In addition, the use of the same methodologies allowed to compare the old map to the new which grant the opportunity to establish what variable has a mayor level of 
influence in landslides hazard.

In the software and geoprocessing section some processes are considered explaining that GRASS was an optimal software in solving many issues presented through the project. In the context section some aspects that affect the stability of hillside in Bogota are described. In the methodology section an overview of both methodologies implied in the model is given. The risk management component highlights the dynamic inherent to the hills stability and therefore the requirement to constant monitoring. Contrasting map section set out the criteria used to combine the partial map results. Finally, implications of this study are briefly explained.

\section{SOFTWARE AND GEOPROCESSING}

\subsection{Formats}

Both formats raster and vector were used harmoniously through the study. $1 \mathrm{~m}$ DEM constituted an essential input in updating of geology, hydrological modelling and in the determination of some variables. However, vector format was used for representing the variables as well as the partial results to be combine. In this way, most of the information was given in vector format, thus, not conversion of data was required in most of the cases.

Since it was necessary continuously to verify the partial results obtain when summing up the different features this format was used for addressing the geographical processes. Likewise, it was necessary to check more than an attribute or characteristic of specific areas -whose hazard was already estimated- in order to verify that the method provides proper results.

\subsection{Geoprocessing}

In the project proprietary software and open source software were available. For executing combinations of layers, GRASS was used exploiting its efficient performance. Other processes executed with GRASS are described subsequently.

\subsubsection{Topology fixing}

Since vector format was mainly used, there were topology issues. To overcome them, the topology nature of GRASS GIS was exploited. Automatic corrections of topology errors are possible to be done with the Clean Vector Map module of GRASS. In this way GRASS becomes a useful tool; manual repair would have been done in absence of it. Correction of topological errors should be made carefully. Therefore, after the correction checking on aerial images could be required.

\subsubsection{Smoothing}

Combination of many layers results in small angles and unpleasant forms in some cases, thus, smoothing process was essential. Performance with proprietary software was not optimal since the polygons shape was rounded without conservation of topology, which bring about a dataset of unconnected polygons. For smoothing the final results the Chaiken algorithm from GRASS -which can be found under the Topology Maintenance menu- was implemented yielding quite acceptable results.

\subsubsection{Watersheds basins}

Accomplishment of watersheds delimitation was executed with GRASS, which provided efficient results. Other software can be sensitive when the size of exterior watershed basin is small e.g. $1000 \mathrm{Ha}$. Those watersheds would present an unpleasant delimitation which would not obey the terrain forms.

\subsubsection{Automation}

When automation of processes was necessary e.g. figures production, routines programmed using the ArcPy module from ArcGIS as well as Model Builder models were implemented. These tools are friendly and ease to use.

\section{CONTEXT OF LANDSLIDES IN BOGOTA}

Bogota is located in the geographic centre of Colombia, in a east Andes mountains plateau, to 2630 above sea level, with a 177598 ha area. Its population exceeds 8080734 .

From the physical perspective, Bogota is formed by two important terrain zones. The first one is a flat zone located in the central and north western part where the population majority live. There the terraces and alluvial valleys of Bogota River and its affluent and wetlands are predominant. The second zone is mountainous and frames the city in the south and eastern side. In this zone the East Hills (Cerros Orientales) are important. (Adapted from Comunidad Andina, 2009)

Accordingly, and due to its hydro-meteorological, geological, geotechnical characteristics, the mountainous zones as well as the flat ones are constantly under natural processes of transformation where the terrain forms change because of frequent precipitations, erosive action of water curses and susceptibility of rocks and soils when these are disintegrated and dragged. In addition, the inadequate human interventions, contribute to the occurrence of different type of landslide phenomena and flooding. (Adapted from Comunidad Andina, 2009)

The mentioned phenomena are considered hazardous. The ridges belonging to the Bogota's Hills have a natural susceptibility to the landslides due to its geology and geomorphology. In addition, human intervention has an impact, the operation of quarries as well as the use of territory for construction of houses without the fulfilment of minimal requirements. (Adapted from Comunidad Andina, 2009) 
It is important to mention, that Bogota is - according to the Colombian Code of Seismic-Resistant Constructionslocated in an intermediate earthquake zone. Furthermore, the city has soils with potential zones of seismic waves' amplification, others with liquefaction, others are expansive and some of the constructions are too vulnerable to earthquakes effects.

Bogota's climate has an impact in life of Bogota`s inhabitants and causes important economical losses- The city is situated in the Intertropical Convergence Zone (ITCZ) which has influence in the rainwater regime. The ITCZ leads to two periods of intensive rainfalls: the first one in months of March, April and May; the second one during the months: September, October and November. During these periods there is a significant coincidence in the increase of landslides reports. (Adapted from Comunidad Andina, 2009).

\section{PROCEDURE AND METHODOLOGIES}

Hazard zoning landslide investigations aim to the importance of tackling a combination of methodologies which are supplemented. A complete inventory of processes of instability of study region should be present as well as a completed geomorphologic characterization.

The method used should allow to establish a comparison to the current landslide hazard map, additionally there was existing information that could be upgraded and also, human factor must have been considered. This could be accomplished by mean of using Semiquantitative Stability Evaluation (SSE). This has the disadvantage of being subjective. Hence, an additional method Natural Slopes Methodology (NSM) is used. The process followed to obtain the landslide hazard map of Bogota 2016 is illustrated in figure 1 . The methodology presented here has a geotechnical background, which is not presented in this paper. In this paper only a very short description will be presented, which does not reflect the potential of these methodologies.

\subsection{SSE Methodology}

The SSE methodology is a heuristic method based on categorizing and weighting of factors causing instability according to the expected influence of these in the occurrence of landslides.

The methodology uses eight variables grouped in 2 categories, the first one is related to the intrinsic conditions of terrain (susceptibility factors): material, relief, drainage and land-use, the second group associated to triggering factors: climate, earthquake, erosion and human factor. (See figure 3).

A layer that depicts each variable is configured, in which variability intervals are assigned according to its influence to landslides stability. To study and retrieved a map from each variable it was necessary to visualise, edit and geoprocess diverse geographic information, the free and Open Source QGIS was used.

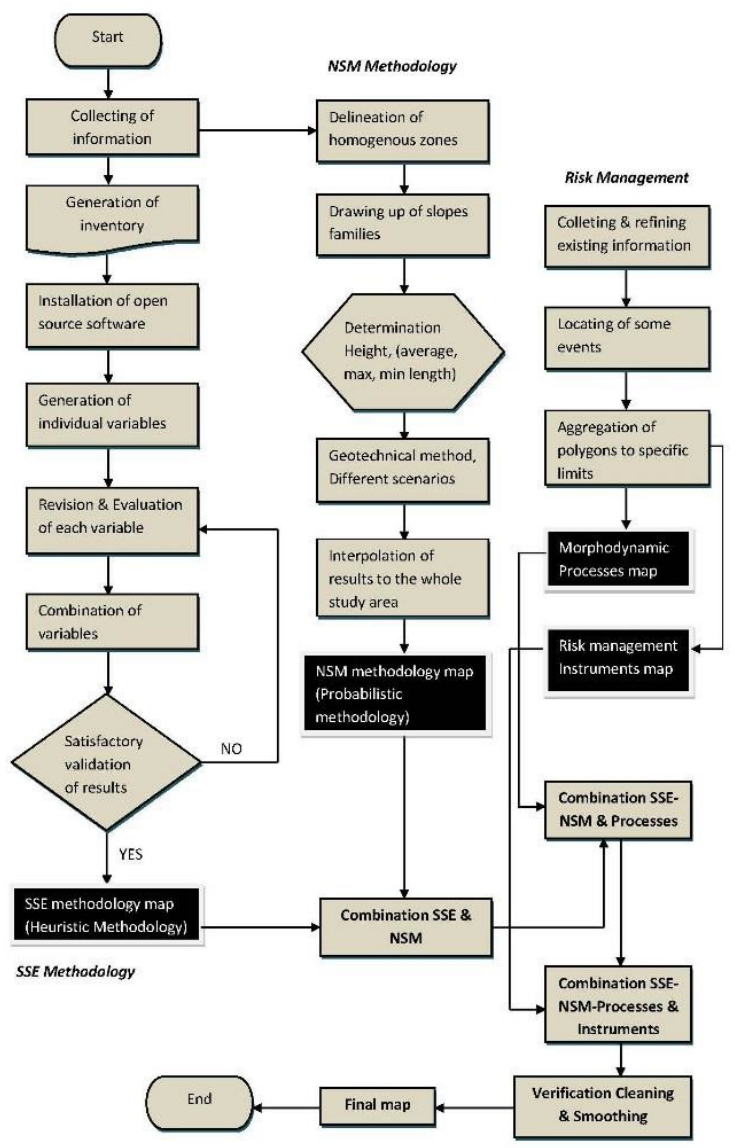

Figure 1 Landslides Hazard Map Procedure
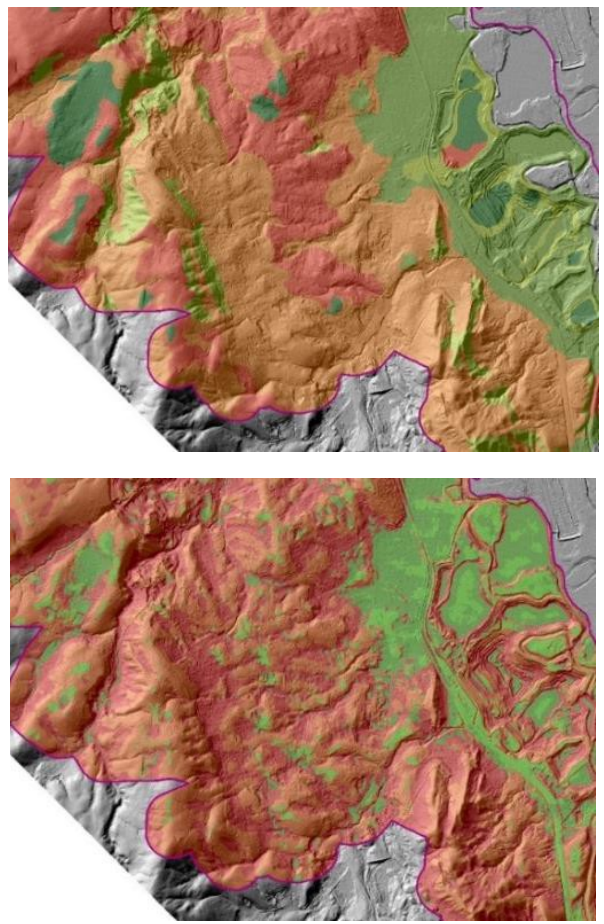

Figure 2 Relief variable comparison (a) 1998 study (b) 2016 study 


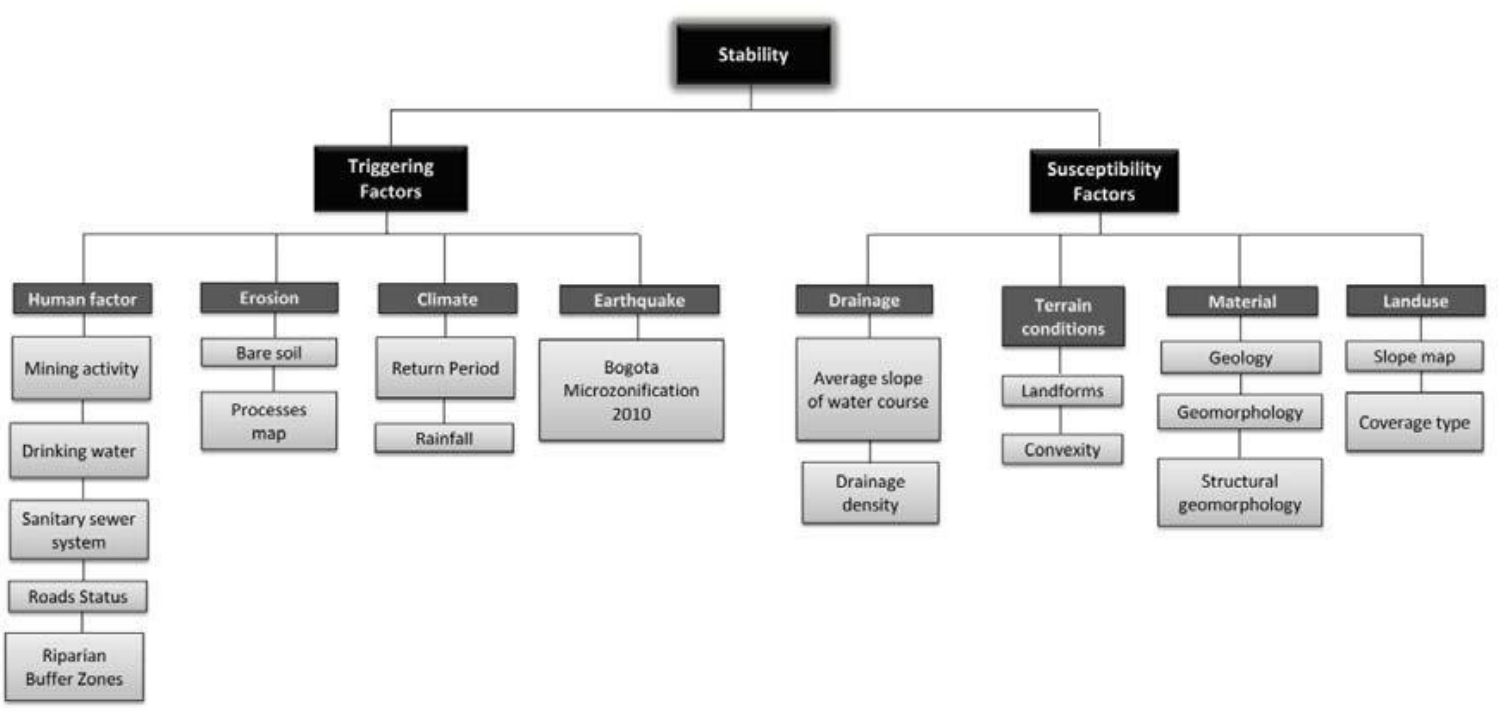

Figure 3 Variables of SSE Method

\subsubsection{Final result of SSE methodology}

Combination of the different factors in each parameter and the sum of the rating from each one, result in a stability rating (SR) from where a level of relative hazard can be defined. Stability can be expressed as the sum of ratings

\section{SR=Human Factor +Erosion +Climate \\ + Earthquake +Drainage +Relief +Material + Landuse}

For rating stability of hillside the SSE approach states that the better the stability is, the higher its rating is. Therefore, the different factors that affect each variable were analysed (See figure 2). The variables were rated with a scale between 1 and 35. For example, for the variable drainage, average slope of water course and drainage density were studied in the hillside area. In the zones where the slope are steep and there is abundant presence of water streams, a very low SR was assigned.

Higher inputs resolution and new available information allowed to improve different variables. Here are described some improvements that had been done as the methodology was applied:

(a) Updating of geology was mainly based on a $1 \mathrm{~m}$ accuracy DEM which allowed to differentiate with a very deep level of detail lithological units as well as the identification of key faults.

(b) Stability conditions are associated to morphometric characteristics and morphodynamic processes. In the analysis, slope as well as convexity were necessary to evaluate. To get relief variable the algorithm Terrain Surface Classification from SAGA GIS was used. The difference in relief variable is evident -when comparing with this one from the previous studysince the landforms obtained with the algorithm depicts the terrain landforms with a much higher level of detail. In where the redder a landform is, the smaller its SR is. (See Figure 2)

The result of combining triggering with susceptibility factors give a final stability result, where the rating were made based on probabilities based on a normal distribution (Figure 4). Important to note is that the higher a value is, the better is its behaviour.

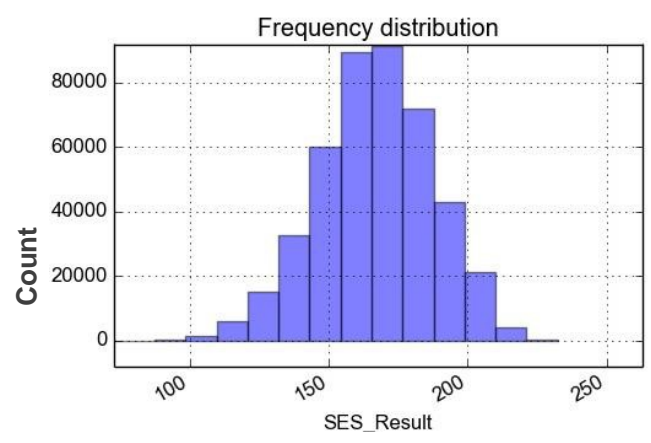

Figure 4 Frequency distribution of SSE methodology result

The results for categorizing the SSE method are shown in table 1.

\begin{tabular}{|l|l|r|r|c|}
\hline Category & Symbol & $\mathbf{1 9 9 8}$ values & 2016 values & Ranges \\
\hline Very high & VH & 126 & 125,4 & SR $<125,4$ \\
\hline High & H & 151 & 146 & $125,4<$ SR $<146,0$ \\
\hline Medium & M & 157 & $\mathbf{1 6 6 , 7}$ & $146,0<$ SR $<187,3$ \\
\hline Low & L & 177 & 187,3 & $187,3<$ SR $<207,9$ \\
\hline Very low & VL & 202 & 207,9 & SR $>207,9$ \\
\hline
\end{tabular}

Table 1 Stability Rating of SSE methodology, previous and updated result 


\subsection{NSM Natural Slopes Methodology}

The basic principle of NSM is that in a family of measurement of heights $(H)$ in meters and their correspondent lengths $(L)$ in meters of vertical gradient lines in a natural hillslope of homogeneous composition and origin are linked by the equation (Taken from Landslide hazard evaluation for Bogota, Colombia):

$$
H=A L^{b}
$$

Where $\mathrm{H}$ and $\mathrm{L}$ are height and length respectively. A and $\mathrm{b}$ are constants in a power function.

Parameter b expresses convexity of the region.

Homogeneous zones are the starting point for determining the slope families. In each homogeneous zone is required to estimate a dataset of heights and lengths of slopes to establish a potential type regression which define each family. The delimitation is a crucial task in the methodology. An acceptable family definition, represents excellent performance in the potential regression correlation with a value of $\mathrm{R}>0.95$.

In the previous study delimitation of slopes families was achieved through the expertise from a specialist, which define the homogenous areas and the slope families in paper maps, in this new study several modifications had taken place:

(a) It is know that the reliability of NSM lies strongly on the accuracy of topographic maps and in the relationship of this accuracy with the relief features. Here the updated geology played an important role since a family of slopes should not intersect more than 1 lithological unit

(b) To define homogenous areas hydrological modelling was applied by mean of defining small watershed units which in turn would be intersected with the synthetic rivers or Accumulation Rivers representing as a result uniform zones. This micro watersheds were related to the updated geology representing very homogeneous areas where slope families could be represented.

Despite this method provides homogeneous areas, after evaluating the regression for each family, some were discarded because of $\mathrm{R}<0.95$, which emphasized again how sensitive is the method and how important is an adequate delimitation process.

Once the similar zones are defined the measurements should fulfil the following requirements (See figure 5):

- The measurements start in inferior contour line.

- The interval between the inferior contour and the next one located immediately in superior level should be measured taking perpendicular distances between both intervals.

- Afterwards, the arithmetic average, maximal and minimal distances should be measured.

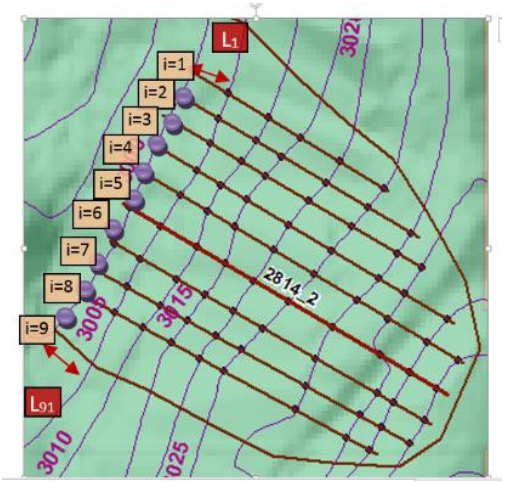

Figure 5 Slope family and its distances

- $\quad$ For the next measurement the starting point is again the inferior contour line, but this time two contour intervals should be covered. For each family all the intervals should be covered.

The assignment described above for taking the measurements would be cumbersome if it was made manually since the families have different sizes and covered different number of contour lines. Therefore, an application in Visual Basic was programmed: initially the coordinates for yielding the distances were separated by family, using a model that iterates and groups according to the name's family.

These groups of coordinates would the input in the VB application. The principle for determining the distances is illustrated in figure 4. Average distance was calculated with the following equation:

$$
\mathrm{L}_{5}=\sum_{\underline{i=1} \mathrm{~L}_{\mathrm{ir}}}^{\mathrm{n}}
$$

$\mathrm{L}_{5}=$ Average length in a $5 \mathrm{~m}$ height

$\mathrm{Lir}_{\mathrm{i}}=$ Distance from the inferior contour intersections (those represented in purple in the figure 4) to the next contour intersections (First measurement)

$\mathrm{n}=$ Number of slopes that formed a family, in this case $n=9$

To find the distances the Euclidean distance formula was used:

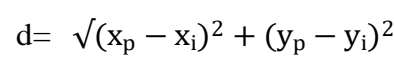

Where $\mathrm{x}_{\mathrm{p}}$ e $\mathrm{y}_{\mathrm{p}}$ are the inferior contour coordinates, $\mathrm{xi}$ e yi are the analysed contour coordinates. $d=$ distance

The table 2 shows the result for the slope family illustrated in figure 5 -It would have 9 lengths-. Here is evident the utility of the VB application since this family has 9 slopes which intersect with the contours 79 times. 


\begin{tabular}{|l|l|l|l|l|}
\hline $\begin{array}{l}\text { H- } \\
\mathbf{2 8 1 4}\end{array}$ & $\begin{array}{l}\text { Average- } \\
\mathbf{2 8 1 4}\end{array}$ & $\begin{array}{l}\text { Maximum- } \\
\mathbf{2 8 1 4}\end{array}$ & $\begin{array}{l}\text { Minimum- } \\
\mathbf{2 8 1 4}\end{array}$ & $\begin{array}{l}\text { Length- } \\
\mathbf{2 8 1 4}\end{array}$ \\
\hline 5 & 7,33356147 & 8,98440664 & 5,44836937 & L1 \\
\hline 10 & 14,1675147 & 16,0439931 & 12,5635029 & L2 \\
\hline 15 & 19,8772509 & 23,0041568 & 18,253848 & L3 \\
\hline 20 & 27,4256795 & 31,3201408 & 24,2235138 & L4 \\
\hline 25 & 35,0314211 & 40,6715744 & 29,0876877 & L5 \\
\hline
\end{tabular}

Table 2 Measurements summary for family 2814

Afterwards, these values are given as inputs in a geotechnical program and some processes are executed where a set of different scenarios like seismic effect, rain plus seismic, and different periods of time (long, medium and short) are analysed. This procedure aims to get Safety Factors (SF) that evaluates the slope stability of each family. In the total scenario a total SF is found and an interpolation map is derivated.

\begin{tabular}{|l|r|}
\hline \multicolumn{1}{|c|}{ SF } & \multicolumn{1}{c|}{ Values } \\
\hline Minimum & 0.15733952 \\
\hline Mean $-1 \sigma$ & 0.38578301 \\
\hline Mean & 0.97912966 \\
\hline Mean+1 $\sigma$ & 1.5724763 \\
\hline Maximum & 3.56908764 \\
\hline
\end{tabular}

Table 3 Intervals for NSM hazard level selection

\begin{tabular}{|l|l|l|}
\hline $\begin{array}{c}\text { MTN } \\
\text { Hazard }\end{array}$ & \multicolumn{1}{c|}{$\mathbf{1 9 9 8}$ Values } & \multicolumn{1}{c|}{$\mathbf{2 0 1 6}$ Values } \\
\hline VH & total_SF $\leq 0.60$ & total_SF $\leq 0.50$ \\
\hline H & $0.6<$ total_SF $\leq 1.1$ & $0.5<$ total_SF $\leq 1.1$ \\
\hline M & $1.1<$ total_SF $\leq 1.9$ & $1.1<$ total_SF $\leq 1.7$ \\
\hline L & $1.9<$ total_SF $\leq 3.4$ & $1.7<$ total_SF $\leq 2.3$ \\
\hline VL & total_SF $>3.4$ & FS_total $>2.3$ \\
\hline
\end{tabular}

Table 4 Levels of hazard NSM methodology

From the safety factors, statistical parameters are obtained. (See Table 3). The values to define hazard intervals are shown in table 4.

\subsection{Risk management}

Apart from these to partial results SSE and NSM, risk management component was in addition considered in landslide map preparation. Instruments and processes were included.

\subsubsection{Instruments}

The risk management instruments include different types of technical studies which evaluate hazard in a detailed way and are used for improving and updating the given information in the normative plan (POT).Thus, for assessing hazards the starting point is the hazard given in the landslide map. Additionally, existing information from previous studies and diagnoses accomplished in the area should be collected.
Relevant information can cover geological units, geomorphology, active or potential processes morphodynamic processes, and drainage characterization. Furthermore, information regarding to land use, and triggering factor like precipitation, human factor (cut and fillings, runoff and superficial water management) and seismicity is included.

Field work should be done to verify physical conditions of the sector under study. There, landslides susceptible zones are delimitated and finally the hazard is defined. These are then inputs in the upgrading of the landslide map.

Since the risk management instruments is generated to provide a mayor level of detail, it is found that the scale of the existing instruments were not compatible with the scale of the landslides hazard plan (1:5000). The detailed concepts can be yielded in a 1:500 or 1:1000 scales, thus, the input entities could not be depicted individually. To reduce the scale of these polygons a concave hull can be drawn around them.

\subsubsection{Processes}

For preparing a processes inventory the initial point is to evaluate the morphodynamic processes; those which are active are considered to have high hazard. Depending on the identified activity from each process, a qualitative probability was assigned, as is shown in the table 5 .

\begin{tabular}{|c|c|}
\hline Process & Probability \\
\hline $\begin{array}{c}\text { Active } \\
\text { process }\end{array}$ & Very high \\
\hline $\begin{array}{c}\text { Incipient } \\
\text { process }\end{array}$ & High \\
\hline $\begin{array}{c}\text { Colapsed } \\
\text { process }\end{array}$ & Medium \\
\hline $\begin{array}{c}\text { Stabilised } \\
\text { process }\end{array}$ & Low \\
\hline
\end{tabular}

Table 5 Hazard according the probability of processes

\subsection{Contrasting of maps and models}

Having obtained the inputs: hazard maps for the SSE and NSM methods with 5 categories of hazard (Tables 1 and 4), map of instruments of risk management with 3 categories (high, medium, low) and processes map with 5 categories, the following procedure was executed:

a. Elaborating of different overlapping models with different levels of conservatism having into account the intervals to define the hazard levels in tables 1 and 4 (previous and new studies values).

In the previous study the validation process was made through visual contrasting between the different partial results in the computer screen and the translucent paper copies of different maps, which is cumbersome and time consuming task and could lead to errors. In the present study this issue was addressed thought performing the following tasks: 
At the beginning the matrix 1 (See table 6) -that overlaps SSE and NSM-was tested where Very high hazard (VH), High (H), Medium (M), Low (L) and Very low (VL). However when the results were validated with the processes map the adjustment was not satisfactory. Likewise a second adjusted matrix was applied. (See table 6 matrix 2).

b. Calibration: This process was possible with the processes whose hazard was known.

A model builder that iterates through all the morphodynamic proceses was configured.The process calculated the percentage of coincidence between processes' hazard levels shown in table 5 and the hazard yielded by the combination SSE+NSM. Such process was posible as the results of the both methodologies as well as the morphodynamic processes were presented in vector format. Four combinations were resulting, from them the more appropiate combination was chosen.

- $\quad$ SSE with 1998 intervals +NSM with 1998 intervals

- $\quad$ SSE with 1998 intervals +NSM with 2016 intervals

- $\quad$ SSE with 2016 intervals +NSM with 1998 intervals

- SSE with 2016 intervals +NSM with 2016 intervals

The results of applying the matrices 1 and 2 were executed for each one of the named combinations to find this one with the best level of adjustment, this by mean of the procedure developed in model builder. The model allowed to register the adjusment of each morphofynamic process and the hazard level derived from each one of the combination listed above. Emphasis was made in the high hazard level polygons, a higher percentage of coincidence was the criterium to decide the winner combination

Once the resulting overlapping maps from performing the matrix 2 were depicted, it was found that for slopes $<10^{\circ}$ and $>45^{\circ}$, the overlapping did not provide an acceptable performance. NSM methodology is applicable in zones different from this range. This is a limitation of the methodology which currently is being studied. Consequently, for this slope range the matrix 3 was applied (table 7), in which the SSE methodology has preponderance. The resulting map after applied this matrix is illustrated in figure $7 \mathrm{a}$.

Afterwards, the processes layer was overlapped with the selected combination NSM-SSE -this that yielded a better level of coincidence with the processes- for evaluating the result the matrix 4 was used. (Table 7)

The result from matrix 4 was then overlapped with the instruments of management risk. Since the processes having high hazard must have entire predominance over the whole information, the result NSM-SSE-ProcessesInstruments was then overlapped with high hazard processes returning the final landslide hazard map of Bogota (Figure 7b)

\begin{tabular}{|c|c|c|c|c|c|c|}
\hline \multirow{1}{*}{} & \multicolumn{6}{|c|}{ SSE } \\
\cline { 2 - 7 } & CAT & VH & H & M & L & VL \\
\cline { 2 - 7 } & VH & VH & VH & H & H & M \\
\cline { 2 - 7 } Z & H & VH & H & H & M & M \\
\cline { 2 - 7 } & M & H & H & M & M & L \\
\hline & L & H & M & M & L & L \\
\cline { 2 - 7 } & VL & M & M & L & L & VL \\
\hline
\end{tabular}

(1)

\begin{tabular}{|c|c|c|c|c|c|c|}
\hline & \multicolumn{6}{|c|}{ SSE } \\
\hline & CAT & VH & $\mathbf{H}$ & $M$ & L & VL \\
\hline \multirow{5}{*}{$\sum_{\mathbf{Z}}$} & VH & VH & $\mathrm{H} 1$ & $\mathrm{H} 2$ & M1 & M2 \\
\hline & H & $\mathrm{H} 1$ & $\mathrm{H} 2$ & M1 & M2 & M3 \\
\hline & M & $\mathrm{H} 2$ & M1 & M2 & M3 & L1 \\
\hline & $\mathbf{L}$ & M1 & M2 & M3 & L1 & L2 \\
\hline & VL & M2 & M3 & L1 & L2 & VL \\
\hline
\end{tabular}

(2)

Table 6 Matrix 1 Conservative Matrix SSE-NSM Matrix 2 Adjusted Matrix SSE-NSM

\begin{tabular}{|c|c|c|c|c|c|c|}
\hline \multirow{7}{*}{ 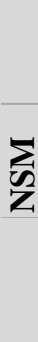 } & \multicolumn{6}{|c|}{ SSE } \\
\hline & CAT & VH & H & $M$ & L & VL \\
\hline & $\mathrm{VH}$ & $\mathrm{VH}$ & $\mathrm{H}$ & $M$ & 珢 & $\mathrm{VL}$ \\
\hline & H & $\mathrm{VH}$ & $\mathrm{H}$ & $M$ & L & $\mathrm{VL}$ \\
\hline & $M$ & $\mathrm{VH}$ & $\mathrm{H}$ & $M$ & $\mathrm{~L}$ & $\mathrm{VL}$ \\
\hline & $\mathbf{L}$ & $\mathrm{VH}$ & $\mathrm{H}$ & $M$ & L & $\mathrm{VL}$ \\
\hline & VL & $\mathrm{VH}$ & $\mathrm{H}$ & $M$ & L & $\mathrm{VL}$ \\
\hline
\end{tabular}

(3)

\begin{tabular}{|c|c|c|c|c|c|}
\hline \multirow{7}{*}{ 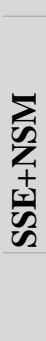 } & \multicolumn{5}{|c|}{ PROCESSES } \\
\hline & CAT. & VH & $\mathbf{H}$ & $\mathbf{M}$ & L \\
\hline & VH & $\mathrm{VH}$ & VH & VH & VH \\
\hline & H & VH & $\mathrm{H}$ & $\mathrm{H}$ & $\mathrm{H}$ \\
\hline & $M$ & $\mathrm{VH}$ & $\mathrm{H}$ & $\mathrm{M}$ & M \\
\hline & L & VH & $\mathrm{H}$ & $M$ & $\mathrm{~L}$ \\
\hline & VL & $\mathrm{VH}$ & $\mathrm{H}$ & $\mathrm{M}$ & L \\
\hline
\end{tabular}

(4)

Table 7 Matrix 3 NSM in $10^{\circ}>$ slopes $>45$

Matrix 4 SSE-NSM \& processes map algebra

\section{IMPACT AND RESULTS}

Landslide hazard levels resulting from the new map are depicted in figure 6 . The graphic illustrates dominance of the level of hazard type medium with $64 \%$, which indicates that for a considerable part of the studied area more specific studies must be developed when a construction 
project

is
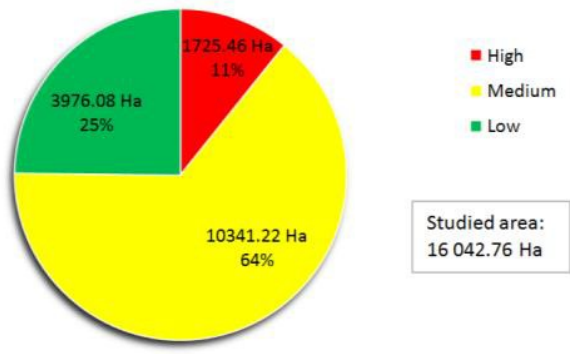

Figure 6 Landslides hazard map

This map has an impact in development of Bogota. It is said that risk management is development management. From this map, new suitable areas for housing projects can be defined. When a project is located in low landslide hazard, specific zone's studies are not needed.

Success of risk management depends on continuous monitoring of likely zones to suffer a landslide. Special attention should be paid to areas characterized in the map as high and medium threats. Since stability conditions are based on diverse triggering and susceptibility factors, the hazard levels are dynamic and new specific studies should be preceded. Hence, this map cannot be interpreted as a definitive map of landslides hazard

When comparing with the previous map Changes in hazard levels can be attributed to: Updating in inputs like seismic acceleration periods (input used in earthquake variable) or inclusion of new climatic stations (input used in Climate variable) could bring about a hazard level can suffer improvement (for example high to medium) or worsening (low to medium).

In addition, reforestation and clean of colluvial deposits could have contributed to improve conditions. Inclusion of risk management instruments could have generated more precise results regarding to hazard zoning.

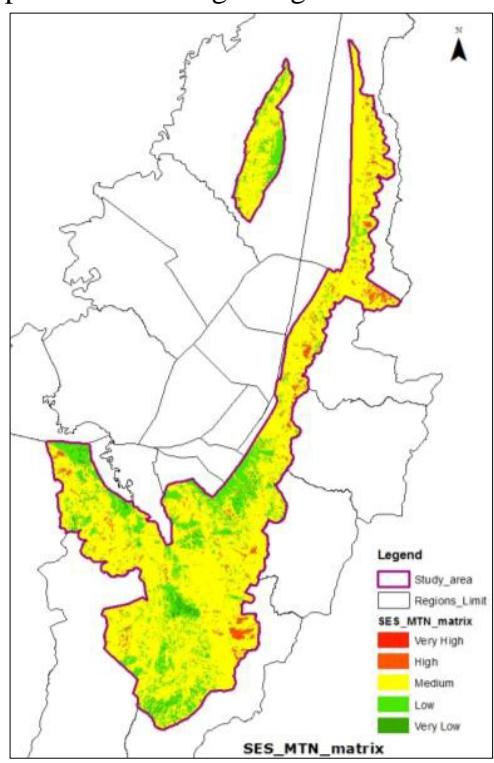

Mitigation measures have an influence in hillside changing geomorphology when housing projects are constructed which improve existent conditions. In addition, human factor plays an important role in stability of slopes, changing the previous conditions.

\section{CONCLUSIONS}

The SSE approach has inherent subjectivity criteria because of weighting assignation. To compensate this flaw the probabilistic approach NSM was used, retrieving quite acceptable results when both approaches are combined. Validation of the results was based on the morphodynamic processes whose hazard has been studied in detail and were known beforehand.

The landslide hazard map helps to have in a quick and easy way a glimpse about the level of hazard in a location according to the map scale production 1:5000. Conversely, specific and detailed studies should been developed when a finding is detected in monitoring process. Moreover, such studies should be conducted when a construction project will be executed.

In processing of large volumes of geographical information other alternatives different from propietary software should be explored since in many cases they offer a better performance e.g. GRASS GIS. Although GRASS GIS is arcane its power makes it worth to be used.

It cannot be expected that the resulting location of the different hazard zones retrieved in a model is infallible. It should be have into account that a model means a simplification of the reality and in this process is possible that some variables cannot be modelled.

In addition, special attention should be paid to the scale 1:5000 which means that the minimal unit to be included in cartography was $400 \mathrm{~m}^{2}$; thus, features smaller that this value were discarded.

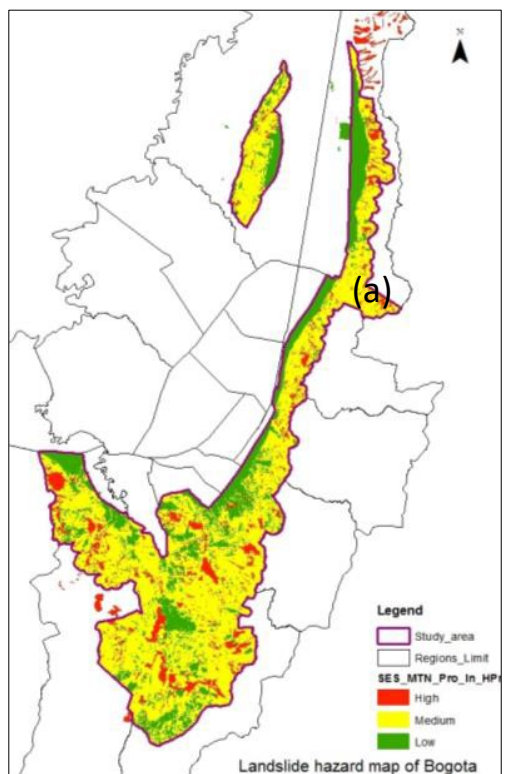

Figure 7 (a) SES_MTN combination using matrix 2 (Table 6), (b) Final approach after combining SES, MTN, Processes, Instruments and verifying high hazard processes 


\section{REFERENCES}

Alcaldía Mayor de Bogotá D.C. (2010). Decreto 523 de 2010. Microzonificación Sísmica de Bogota D.C.

Comunidad Andina. (2009). Prevención y reducción de riesgos a través de los instrumentos de planificación territorial en Bogotá. Lima

Cruden, D. M., \& Varnes, D. J. (1996). Landslide types and processes. In K. Turner \& R. L. Schuster (Eds.), Landslides investigation and mitigation: Washington D. C, National Academy Press, Transportation Research Board Special Report 247 (pp. 36-75).

Dirección de Prevención y Atención de Emergencias DPAE. (2006). Documento Técnico de Soporte - Plan Distrital de de Prevención y Atención de Emergencias PDPAE. Bogotá, D.C.

Dirección de Prevención y Atención de Emergencias DPAE. (2008). Instrumentos para la gestión del riesgo en Bogotá. Bogotá, D.C.

GRASS Development Team, 2017. Geographic Resources Analysis Support System (GRASS) Software, Open Source Geospatial Foundation http://grass.osgeo.org (20 September 2017)

Hungr, O. (2016). A review of landslide hazard and risk assessment methodology. In S. Aversa, L. Cascini, L.

IDIGER. (2016). Diagnóstico para Fase Preliminar Proyecto de Actualización del Componente de Gestión del Riesgo para la Revisión Ordinaria del Plan de Ordenamiento Territorial de Bogotá D.C. Bogotá D.C.

IDIGER. (2016). Amenaza por movimientos en masa documento técnico de soporte. Actualización del POT

Picarelli, \& C. Scavia (Eds.), Landslides and Engineered Slopes. Experience Theory and Practice Proceeding of the 2th International Symposium on Landslides (pp. 3-27). Napoli, Italy.

Shuk E., T. (1990). La Evolución y el Estado Actual de la Metodología Basada en Taludes Naturales para Análisis de Estabilidad en Masas de Materiales Geológicos. In Parte IIII - CSMR—SVMSIF. Caracas.

Soeters, R., \& Van Westen, C. J. (1996). Slope instability recognition, analysis and zoning landslides Investigation and Mitigation. In K. Turner \& R. L. Schuster (Eds.), Landslides Investigation and Mitigation; Transportation Research Board, Special Report 247 (pp. 129-177). Washington D. C.: National Academy Press. 